Rainer Bayreuther (Lüneburg)

\title{
Die Rhythmusbewegung im frühen 20. Jahrhundert und ihre Grundlegung in der empirischen Ästhetik
}

Oft wurde bemerkt, Kant habe seine Theorie vom apriorischen Anschauungscharakter von Raum und Zeit erdacht, ohne auf nennenswerte historische Vorläufer zurückgreifen zu können. Parallelen in der ästhetischen Praxis aber hatte er, mindestens beim Zeitaspekt. In der Musik der Wiener Klassik um 1780 etabliert sich eine Zeitordnung der Töne, die in der Musikforschung als „leerer Takt" bezeichnet wird: ${ }^{1}$ eine unausgefüllte Zeitspanne, aber trotz Leere in sich strukturiert durch schwere und leichte Zeitpunktstellen, nach denen sich harmonisch und melodisch ordnet, was der Komponist an Tönen und Tondauern hineinfüllt. Der Mikrokosmos der gefüllten Dauern kann eigener Regel und Willkür gehorchen. Im Großen aber muss er sich der Struktur des leeren Takts fügen. Was den durchbrochenen Satz eines Haydn-Quartetts und eines Sängerensembles einer Mozart-Oper schlüssig erklärt, ist nichts anderes als eine transzendentale Musikästhetik, die auf apriorischen Zeiträumen basiert. Die Zeitstruktur der akustischen Ereignisse im Innern der Zeiträume wird hier immer in Abhängigkeit der Zeiträume selbst erfasst. Vor allem ändert die Zeitstruktur der akustischen Ereignisse im Innern nichts an der Struktur der apriorischen Zeiträume.

Diese Zeitordnung von Tönen und Klängen ist nicht gottgegeben, wie sich im 19. Jahrhundert zeigt. Die Musiker erfinden Klanggebilde, die sich nicht nur keinen leeren Zeitfenstern mehr fügen (etwa der ganz und gar nicht marschmäßige Trauermarsch in Wagners Götterdämmerung), sondern überhaupt keine leere Zeit mehr voraussetzen (etwa die tonmalenden Passagen in Strauss' Symphonischen Dichtungen). Die Musik ergreift offenkundig Möglichkeiten, die es schlechterdings gibt und die in der Eigentümlichkeit der menschlichen Wahrnehmung begründet sind, Zeiträume nicht unabhängig von den Dingen aufzufassen, die sie füllen. Dinge aber sind konkret, keine abstrakten Strukturen. Das ist das bahnbrechende Resultat der empirischen Psychologie der Zeitwahrnehmung, das sich am Ende des 19. Jahrhunderts abzeichnet. „Denn die Zeit kann keine Bestimmung äußerer Erscheinung sein“, sagte Kant. ${ }^{2}$ Doch, kann sie, sagt die empirische Zeitpsychologie. Die künstlerischen Konsequenzen sind enorm. Sie beinhalten namentlich, dass die Zeitbestimmungen, die sich auf den verschiedenen Feldern der akustischen, visuellen, taktilen Sinneswahrnehmung ergeben, permanent ineinander übergehen. Sie beinhalten nebenbei, dass ein tiefliegendes, unspezifisch auf Adorno zurückgehendes Vorurteil klassisch geschulter Musik(wissenschaftl)er, die Rhythmik der Popmusik sei trivial, weil sie immer auf das simple durchgeschlagene Zweiermetrum reduzierbar sei, hinfällig wird: Welchen Groove ein Stück im binären Beat hat, wie also der binäre Beat selbst wahrgenommen wird, entscheidet sich nicht am binären Beat, sondern an seiner rhythmischen Ausfüllung. (An diesem Faktum setzt die Respondenz Großmanns an.) Das Scharnier zwischen den Sinnesfeldern ist Bewegung, oder ästhetisch gesagt: Rhythmus. So eröffnet sich um 1900 ein

1 Thrasybulos Georgiades, „Aus der Musiksprache des Mozart-Theaters“, in: Mozart-Jahrbuch 1950, S. 76-98.

2 Immanuel Kant, Kritik der reinen Vernunft (A: 1781, B: 1787), A 33/B 49. 
Feld synästhetischer Zeitkünste, systematisch ausformuliert durch neue Zeitästhetiken und -philosophien, pädagogisch ausbuchstabiert in der Reformpädagogik.

Die Akteure der Rhythmusbewegung im frühen 20. Jahrhundert kommen aus verschiedenen Sparten. Beteiligt sind Künstler: Musiker, Dichter, Tänzer, Maler; Wissenschaftler: Philosophen, Psychologen, Germanisten, Musikwissenschaftler, Sportwissenschaftler; Pädagogen: Musikpädagogen, Sportpädagogen, Reformschulmänner, ehrenamtliche Führer von Gruppen der Jugendbewegung, politische Pädagogen; Funktionäre der Kulturpolitik, der Bildungspolitik, des Sports. Die Transdisziplinarität des Phänomens Rhythmus ist seitdem erhalten geblieben, hat sich mittlerweile aber so diversifiziert, dass die meisten Autoren nurmehr unbewusst dessen geschichtliche Anfänge fortschreiben. ${ }^{3}$ So erklärt sich, dass Rhythmus zur Großen Metapher umfassender Welterklärungen avancieren konnte, angefangen bei Klages, ${ }^{4}$ nicht endend bei Deleuze/Guattari. ${ }^{5}$

Weder bilden die Akteure des frühen 20. Jahrhunderts ein großes personelles Netzwerk aus noch sind sie von denselben philosophischen, psychologischen und ästhetischen Prinzipien geleitet. Es gibt Verflechtungen, aber auch Abgrenzungen, Feindschaften, Nebeneinander. Nicht wenige, aber nicht alle Akteure entwickeln ihre Vorstellung des Rhythmischen aus direkter Beschäftigung mit der empirischen Ästhetik des späten 19. Jahrhunderts. Teilweise übernehmen sie die Erkenntnisse der empirischen Ästhetik aus zweiter oder dritter Hand. Bei wieder anderen kann man pauschal nur sagen, dass eine gemeinsame Aversion gegen abstrakte ästhetische und wissenschaftliche Konzepte besteht und ein möglichst unmittelbares und eigenes künstlerisches Erfahren in den Mittelpunkt rückt. Dennoch koinzidieren die Auffassungen und Aktivitäten zum rhythmischen Erleben in bemerkenswerter Weise. Das berechtigt, von einer Rhythmusbewegung zu sprechen in dem Sinn, dass die Frage des rhythmischen Erlebens im frühen 20. Jahrhundert eine Sog- und Sammlungskraft über personelle, disziplinäre und weltanschauliche Grenzen hinweg entfaltete. In den persönlichen und disziplinären Netzwerken, Feindschaften und Ignoranzen der wichtigsten Akteure der Rhythmusbewegung, die ich der Reihe nach - und oft nur skizzenhaft - durchgehe, kristallisiert sich hier verschwommener, dort klarer, aber letztlich einmütig die Behauptung: Rhythmus ist, im Unterschied zum Metrum, rein phänomenal gegeben. Er ist weder eine Eigenschaft der Wahrnehmung noch eine der Dinge an sich, sondern eine der Wahrnehmungsbewegung, die der Dinge bedarf, um sich vom einen zum anderen Ding rhythmisch weiterzubewegen. Wie das vor sich geht und warum die rhythmische Bewegtheit der Wahrnehmung das Primäre ist und ein an den Dingen festgestelltes Metrum das Sekundäre, sagt um 1900 in dieser Klarheit erstmals die empirische Ästhetik.

3 Weitgefächerte Belege von vor 1900 bis in die Gegenwart bei Jean-Claude Schmitt, „Eine Geschichte der Rhythmen: warum und wie?"“, in: Rhythmus - Balance - Metrum. Formen raumzeitlicher Organisation, hrsg. von Christian Grüny und Matteo Nanni, Bielefeld 2014, S. 15-30.

4 Ludwig Klages, Der Geist als Widersacher der Seele, Leipzig 1929.

5 Gilles Deleuze und Félix Guattari, Mille Plateaux, Paris 1980, bes. Abschnitt 11 über das Ritornell. 


\section{Rhythmusforschung in der empirischen Ästhetik}

Über Herbart, ${ }^{6}$ Mach $^{7}$ und Fechner $^{8}$ rückt im 19. Jahrhundert die menschliche Zeitwahrnehmung allmählich in den Fokus der Psychologie. Nach und nach kristallisieren sich die zentralen Fragen: Welche Minimal- und Maximaldauer hat die Aufmerksamkeitsspanne des menschlichen Bewusstseins? Wie wirken die Dauer der Aufmerksamkeitsspanne und ihre Binnengliederung durch akustische (oder optische oder taktile) Reize im Wechsel? Wie untergliedert das Bewusstsein diese Gliederung in sich? Welche Rolle spielt dabei die Periodik der Gliederung? Wie kommt Periodizität überhaupt zustande? Erfolgen solche Gliederungen mono- oder synästhetisch? Nach und nach wird auch den musikaffinen Theoretikern klar, dass sich diese Fragen nicht durch musikalische Analyse lösen lassen, sondern die Musik nur Spezialfall einer umfassenderen Psychologie des Zeitbewusstseins ist. Nach und nach schließlich wird deutlich, dass sich die Fragen im Detail nicht durch Überlegung, sondern nur durch Experiment beantworten lassen. Die jüngere empirische Ästhetik der Jahrhundertwende geht daher zu Experimenten über, paradigmatisch in Hermann von Helmholtz' Lehre von den Tonempfindungen. ${ }^{9}$ Helmholtz gelangt zu völlig neuen Erkenntnissen in der Wahrnehmung einzelner (Ton) und zusammengesetzter (Akkord) Klänge und deren Konsonanzgrad zueinander. Daraus ergibt sich eine neue Grundlage für die Lehre der Klangverbindungen. Aber Helmholtz lässt die zeitliche Gliederung von Klangfolgen außer Betracht, ein schwerwiegendes methodisches Manko, wie sich bald zeigen wird, denn nicht nur haben die Konsonanzqualitäten der Töne und Klänge Einfluss auf Dauer und Binnengliederung von Aufmerksamkeitsspannen, sondern umgekehrt haben Dauer und Binnengliederung Einfluss auf die Wahrnehmung der Tonqualitäten selber. Die Dimensionen sind verschränkt. Das ist die bahnbrechende Einsicht, zu der erst Wilhelm Wundt und seine Schüler, und auch diese erst allmählich, gelangen. In den ersten drei Auflagen von Wundts Grundzügen der physiologischen Psychologie ${ }^{10}$ findet sich eine Reihe von Überlegungen zu Dauernfolgen: Maximal wie lange bzw. wie kurz darf eine (akustisch begrenzte) Einzeldauer innerhalb einer Folge sein, damit sie als distinkte Dauernentität wahrgenommen wird? Wie viele solcher (annähernd) gleicher Dauern können Inhalt eines Bewusstseinsgehalts sein, von denen der Proband die Anzahl nennen kann, ohne mitgezählt zu haben? Das sind wichtige erste Ansätze der experimentellen Dauernwahrnehmung, die aber noch nicht die entscheidende Frage nach der „rhythmischen“ Eigendynamik der Aufmerksamkeit fragen, die in die objektiv gegebenen Sinnesdaten von akustischen Dauern und ihren akustischen Grenzen bestimmte Strukturen hineinträgt. Dies bringt die stark umgearbeitete vierte Auflage (1893), der ganz neue Experimente an Wundts Leipziger „Institut für experimentelle Psychologie“ vorangegangen sind. Unter anderem beschreibt Wundt den elektromagnetischen Schallhammer, ${ }^{11}$ eine Vorrichtung, die Anschläge unterschiedlicher Dynamik und Zeitabstände in absolut identischen Tonqualitäten und Dauernquantitäten produzieren kann. Mit diesem Apparat führt sein Habilitand Ernst Meumann die Experimente durch, mit denen im Wesentlichen zweierlei nachgewiesen wird: Objektiv gleiche Tonqualitäten, die in identi-

\footnotetext{
6 Johann Friedrich Herbart, Psychologische Untersuchungen, Göttingen 1839.

7 Ernst Mach, Untersuchungen über den Zeitsinn des Ohres, Wien 1865, S. $133 \mathrm{f}$.

8 Gustav Theodor Fechner, Vorschule der Ästhetik, Leipzig 1876.

9 Hermann von Helmholtz, Die Lehre von den Tonempfindungen als physiologische Grundlage für die Theorie der Musik, Braunschweig 1863.

10 Wilhelm Wundt, Grundzüge der physiologischen Psychologie, Leipzig ${ }^{1} 1874,{ }^{2} 1880,{ }^{3} 1887$.

11 Ebd., ${ }^{4} 1893$, Bd. 2, S. 423.
} 
schen Zeitintervallen gehört werden, werden als ungleich wahrgenommen. Und objektiv gleich lange Zeitintervalle, die durch ungleiche Tonqualitäten begrenzt werden, werden als ungleich lang wahrgenommen. Beide Formen der akustischen Täuschung unterliegen bestimmten Gesetzmäßigkeiten. ${ }^{12}$ Und beide markieren die Differenz des Rhythmischen (der Phänomenalität der akustischen Ereignisse) zum Metrischen (der objektiven Struktur der akustischen Ereignisse). Es sind diese Experimente, die der späteren Rhythmusbewegung die entscheidenden Impulse geben, wenngleich man sich statt auf Meumann eher auf Wundt beruft. In der Rhythmusbewegung nicht mehr wahrgenommen, weil zu technisch, wird die Studie Psychologie der Zeitauffassung von Vittorio Benussi. ${ }^{13}$ Benussi, Mitarbeiter in Alexius Meinongs „psychologischem Laboratorium“ in Graz, entwickelt die Erkenntnisse Meumanns weiter, und zwar mit einer erheblich ausgeweiteten Anzahl an Versuchspersonen. Viele Befunde Meumanns bestätigt und differenziert er, einige revidiert er.

\section{Philosophie}

In der Philosophie sind Henri Bergson und Ludwig Klages die Ideengeber. Bergson veröffentlicht 1889 den Essai sur les données immédiates de la conscience, in dem er darlegt, die Bewegtheit des Bewusstseins rühre von einer Bewegung der Aufmerksamkeit von einem zum nächsten Objekt. Ist das Bewusstsein auf diese Weise in der Lage, eine raumzeitliche Beziehung zwischen den Objekten herzustellen, ist seine Wahrnehmungsbewegung also kontinuierlich, dann ist sie rhythmisch. Das Rhythmische des Bewusstseinszustands ist folglich eine Zeit- und Raumverhältnisse konstituierende seelische Tätigkeit. Das fasst Bergson zugleich als rhythmische Bewegtheit des Lebens insgesamt auf. Die empirische Forschung zum menschlichen Bewusstsein, namentlich Fechner und Ernst Heinrich Weber, steht im Hintergrund und wird immer wieder angeführt. Vereinzelt wird in Deutschland diese Lebensphilosophie schon um 1900 im französischen Original zur Kenntnis genommen, zum Beispiel von Stefan George und Figuren des sich bildenden George-Kreises, namentlich Norbert von Hellingrath und Ludwig Klages. Verbreitung in Deutschland erfährt erst die deutsche Übersetzung Zeit und Freiheit von 1911, in der Bergson die zwischenzeitlich entstandene Literatur zur experimentellen Ästhetik berücksichtigt, etwa Wundts Grundzüge der physiologischen Psychologie in der sechsten Auflage von 1910. ${ }^{14}$ Im GeorgeKreis wird Bergson eingehend diskutiert, auch im Kontext der Überlegungen zum dichterischen Rhythmus, die Hellingrath in den Kreis einbringt. ${ }^{15}$ Auch Philosophen, die in der Rhythmusbewegung dann viel gelesen werden, sind ihrerseits Bergson-Leser: weiterhin Klages, der Bergsons phänomenologische Analyse der Dauer wie auch den Gedanken

12 Meumanns Habilitationsschrift erschien in drei Teilen: „Untersuchungen zur Psychologie und Aesthetik des Rhythmus", in: Philosophische Studien 10 (1894), hrsg. von Wilhelm Wundt, S. 249-322 (1. Teil) und S. 393-430 (2. Teil), „Beiträge zur Psychologie des Zeitbewusstseins. Dritte Abhandlung“, in: Philosophische Studien 12 (1896), hrsg. von Wilhelm Wundt, S. 127-254 (3. Teil).

13 Vittorio Benussi, Psychologie der Zeitauffassung, Heidelberg 1913.

14 Henri Bergson, Essai sur les données immédiates de la conscience, Paris1889; dt.: Zeit und Freiheit, Jena 1911.

15 Norbert von Hellingrath, Pindarübertragungen von Hölderlin. Prolegomena zu einer Erstausgabe, Jena 1911. Zu der von Hellingrath im George-Kreis angestoßenen Diskussion zum dichterischen Rhythmus vgl. Gunilla Eschenbach, „Wie dichtet der ,Urgeist? Hellingraths Konzept der harten Fügung“, in: Norbert von Hellingrath und die Ästhetik der europäischen Moderne, hrsg. von Jürgen Brokoff, Joachim Jacob und Marcel Lepper, Göttingen 2014, S. 107-118. 
des Lebensrhythmus übernimmt, aber auch der junge Heidegger, der ihn stark transformiert, indem er ihn aus der Thematik der Bewusstseinsakte herauslöst und als existentiale Grundverfasstheit der Ontologie überhaupt behauptet. Lebensbewegtheit ist bei Heidegger keine Eigenschaft von Bewusstseinsakten mehr, sondern das existentiale Fundament, auf dem das menschliche Bewusstsein seine Eigenschaften wie auch Eigenschaften anderer Entitäten bestimmt. ${ }^{16}$ Man sieht hieran, wie sehr Heideggers Existenzphilosophie von der empirischen Wahrnehmungsforschung angeregt ist, sich aber in Konsequenz scharf gegen alles Psychologische abgrenzt. Ebenso ist es inspiriert von der Phänomenologie des inneren Zeitbewusstseins Husserls, Vorlesungen aus den Jahren 1904 bis 1910, die Heidegger genauestens kennt, da er ihre erste Edition 1928 selbst besorgt. ${ }^{17}$ Husserl kennt die empirische Forschungslage, zumal er mit der Meinong-Schule in Graz Kontakt pflegt, stellt aber schon in den ersten Zeilen klar, dass er seine Zeitphänomenologie in kategorialer Unterscheidung zu aller Zeitpsychologie strikt nichtpsychologisch versteht. Sein Argument: Die Zeitphänomenologie nimmt die Phänomenalität der Zeit als solche, ohne nach irgendeiner objektiven Zeitrealität zu fragen. Die Psychologie dagegen fasst das Zeitbewusstsein als psychisches Überformen objektiver Zeitrealität auf. So weit, so richtig, aber Husserl behauptet weiter, nur in einer reinen Phänomenologie können die Gesetzmäßigkeiten des Zeitbewusstseins erforscht werden. Diesem strittigen Argument hätten experimentelle Zeitpsychologen wie Meumann widersprochen. Heidegger jedenfalls treibt die Psychologismuskritik noch weiter als sein Lehrer. Auch mit Klages setzt sich Heidegger auseinander, tut ihn öffentlich aber als Popularphilosophen ab. ${ }^{18}$ Dass wir mit den genannten Philosophen, die die innere Zeitlichkeit von Bewusstseinsvorgängen erforschen, ziemlich genau die bedeutenden Namen der Phänomenologie abgeschritten haben, ist kein Zufall. Die Rhythmusbewegung ist in ihrer Grundhaltung phänomenologisch. Sie gewinnt, von der empirischen Psychologie des Zeitbewusstseins aus- und über sie hinausgehend, die Überzeugung, dass das Rhythmische als Eigenschaft eines Bewusstseinsakts nur unzureichend beschrieben ist. Über den Akt geht es in den Bewusstseinsgehalt über. Genau daran schärfen Husserl und Heidegger ihren zeitphilosophischen Antipsychologismus.

\section{Dichtung}

Bei den Dichtern formuliert als Erster Arno Holz einen Begriff des dichterischen Rhythmus, der sich von der Metrik der erklingenden Worte dezidiert ablöst: Er fordert „eine Lyrik, die auf jede Musik durch Worte als Selbstzweck verzichtet und die, rein formal, lediglich durch einen Rhythmus getragen wird, der nur durch Das lebt, was durch ihn zum Ausdruck

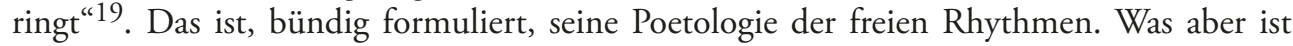
das „Das“? Die Dinge an sich? Die Phänomene? Karl Freiherr von Levetzow wählt in seiner Antwort erstere Lesart. Das mündet erkennbar in einen schlechten Naturalismus, und

16 Ausgearbeitet in Martin Heidegger, Sein und Zeit (1927), Tübingen ${ }^{14} 1977$. Vgl. die frühen Freiburger Vorlesungen und bes. den Vortrag Der Begriff der Zeit (1924, ed. Tübingen 1989), in denen sich Heideggers antipsychologischer Zeitbegriff ausbildet.

17 Edmund Husserl, „Vorlesungen zur Phänomenologie des inneren Zeitbewußtseins“, in: Jahrbuch für Philosophie und phänomenologische Forschung IX (1928), S. 367-498, hrsg. von Martin Heidegger.

18 Martin Heidegger, Die Grundprobleme der Metaphysik (Freiburger Vorlesung WS 1929/1930), GA 29/30, S. 105.

19 Arno Holz, „Selbstanzeige“ (zum Erscheinen des Phantasus), in: Revolution der Lyrik, Berlin 1899, S. 21-24 und S. 26-31, hier S. 22. 
dessen bezichtigt er Holz. Er verteidigt den subjektiven Zugriff des Dichters auf die Welt: „Jeder Künstler aber ist nothwendig subjectiv, Individualist. Es gibt keine objective Kunst. Denn `Ich und die Welt‘, diese Gegenüberstellung ist die letzte Lebenseinheit jeder Kunst. Wo das aufhört, beginnt Nirvana und es ist überhaupt alles zu Ende. [...] Alles Leben, alle Bewegung, aller Rhythmus, alle Kunst hat aufgehört. Deshalb halte ich den Naturalismus in seinem eigentlichen Wesen für einen Fortschritts- und Entwicklungshemmer, für kunstund culturfeindlich. “ ${ }^{20}$ Bergsons „élan vital“ klingt an, ist aber allenfalls vage assoziiert, denn die Unterscheidung zwischen Subjektivität und Phänomenalität, die Bergson schon klar vor Augen steht und in der er das Leben auf die Seite von letzterem schlägt, bleibt hier schwammig. Der weitere Fortgang der Auseinandersetzung zwischen Holz und Levetzow ${ }^{21}$ kreist um diese Problematik, ohne sie zu durchdringen.

All das sind sichere Indizien, dass ohne Kenntnis der empirischen Forschung argumentiert wird. Den empirischen Diskurs voraus setzt hingegen der Zirkel der Kosmiker in Schwabing mit Karl Wolfskehl als Mäzen und Mittelpunkt, Klages, George und andere als Mitglieder. Wer dort das Thema Rhythmus zuerst aufwirft, ist schwer zu sagen. Fakt ist, dass Klages in seinem George-Buch von 1902 mottohaft einen Aphorismus Georges zum dichterischen Rhythmus („maass“) zitiert, den dieser erst 1903 im Prosawerk Tage und Taten $^{22}$ veröffentlicht: „Den wert der dichtung entscheidet nicht der sinn (sonst wäre sie etwa weisheit gelahrtheit) sondern die form $\mathrm{d}$. h. durchaus nichts äusserliches sondern jenes tief erregende in maass und klang wodurch zu allen zeiten die Ursprünglichen die meister sich von den nachfahrenden künstlern zweiter ordnung unterschieden haben. " ${ }^{23}$ Noch im Bann des Schwabinger Zirkels fasst Klages Georges Dichtung bereits mit dem Geist-SeeleDualismus. Georges Gedichtbände Das Jahr der Seele (1897) und Der Teppich des Lebens (1900) werden von Klages mit dem Dualismus gedeutet. Man beachte die Wortwahl in den Titeln: Die Seele als Inbegriff des menschlichen Bewusstseins, das das leibliche und das geistige Moment vereint, als Ort der Phänomene, wird bei Klages später zur Gegeninstanz zur abstrahierenden Verstandestätigkeit, die er Geist nennt; das Leben als noch umfassenderen Begriff verstehen George und dann Klages im Sinn der Bergson'schen Lebensphilosophie als Gesamtheit aller seelischen Akte eines Menschen oder sogar einer Gemeinschaft Mehrerer. Den Unterschied der dichterischen Form - neben den aus Wortklang gebildeten Strukturen vor allem das Metrum - zwischen ursprünglichen Meistern wie George und zweitrangigen Dichtern fasst Klages so: „erlebt - nie begriffen “24. Das Metrum der Dichtung ist keine abstrakt gewählte und abstrakt geltende Form, sondern durchherrscht vom Rhythmus von Seele und Leben. Damit ist der Kern von Klages' gesamtem späterem Theoriewerk fixiert: Wo immer in solcher Kunst ein Sachverhalt ästhetisch aufgefasst wird, wurde er rhythmisch vom Leben gegeben und rhythmisch durch den Künstler erfasst.

20 Karl Freiherr von Levetzow, „Der neue Rhythmus“, in: Die Zeit 5 (1898/99), S. 56-58, hier S. 56.

21 Geführt in den Folgenummern von Die Zeit (1899).

22 Stefan George, „Tage und Taten“, in: ders., Werke Bd. 2, München 2000, S. 530. Klages zitiert in abweichender Orthographie.

23 Ludwig Klages, Stefan George, Berlin 1902, S. 11.

24 Ebd., S. 18. 


\section{Tanz und Gymnastik ${ }^{25}$}

Oft werden nur die Vertreter der Disziplinen Tanz und Gymnastik als Rhythmusbewegung gefasst, eine zu enge Bestimmung der Bewegung, was schon daran erkennbar ist, dass sie ihren Ausgang in der Musik nimmt und später wieder in musikalische Strömungen mündet, deren Ästhetik sich aus dem hier verhandelten Rhythmusbegriff nährt, etwa Carl Orff. Daneben differenziert sie sich aus in sportliche Disziplinen wie Gymnastik, Turnen, Leichtathletik und allgemein sportwissenschaftliche Ansätze. Sie mündet in unterschiedliche Pädagogiken. Sie mündet schließlich in Staatstheorie und Staatspraxis. Sicher aber sind die Tänzer und Gymnasten zwischen der Lebensphilosophie vor 1900 und den Ausdifferenzierungen des Rhythmusdenkens um 1930 die wichtigste Vermittlergruppe.

Der Genfer Komponist und Musikpädagoge Émile Jaques-Dalcroze entwickelt in den 1890er Jahren Methoden, mit seinen Klavierschülern die rhythmischen Strukturen der Musik nicht an den Tasten zu lernen, sondern sie durch Körperbewegungen und bewusstes Atmen auf anderen sinnlichen Ebenen zu erfassen. Die „Methode Jaques-Dalcroze“, weitgehend dokumentiert von seiner Assistentin Nina Gorter, ${ }^{26}$ wird die rhythmische Klavierpädagogik in eine umfassende Theorie der Rhythmuswahrnehmung einordnen. Hintergrund sind nicht die empirischen Ästhetiker, die Jaques-Dalcroze nicht gekannt haben dürfte. Hintergrund ist der Delsartismus, ein auf den Sänger und Schauspieler François Delsarte (1811-1871) zurückgehendes System, das seinerseits aus der Praxis stammt und sich selbst eine popularphilosophische Basis gibt. Auf solidere wissenschaftliche Grundlagen stellen es erst JaquesDalcrozes zahlreiche Schüler. Fast alle durchlaufen sie die „Bildungsanstalt für Musik und Rhythmus E. Jaques-Dalcroze“ in Hellerau bei Dresden, gegründet 1910. Hellerau wird zum Ausgangspunkt für nahezu alle Tanz- und Gymnastikschulen, die sich nach dem Krieg in Deutschland bilden. Sie unterscheiden sich praktisch wie konzeptuell zum Teil erheblich. Der Grund ist, dass trotz der persönlichen Ausstrahlung und des großen Erfolgs, den Jaques-Dalcroze hat, Theoriedefizite spürbar sind, die von den Schülern auf unterschiedliche Weise behoben werden, und zwar unter unmittelbarem oder mittelbarem Rückgriff auf die empirische Ästhetik.

Rudolf von Laban, aufgewachsen im Wiener Adelsmilieu der späten Donaumonarchie, findet über ein Malereistudium in Paris und privaten Ballettunterricht zum Thema Rhythmus. Und er ist ein veritabler Klavierspieler. 1913 kommt er in München mit der Schweizerin Suzanne Perrottet zusammen, einer Assistentin Jaques-Dalcrozes in Hellerau. Er befasst sich mit der „Methode“, kann ihr aber nicht viel abgewinnen, da sie das Rhythmische musikalisch und nicht von der Bewegung her denkt. Im selben Jahr halten sich beide auf dem Monte

25 Vgl. den Umriss der Tanzbewegung bei Gabriele Brandstetter, „Rhythmus als Lebensanschauung. Zum Bewegungsdiskurs um 1900", in: Aus dem Takt. Rhythmus in Kunst, Kultur und Natur, hrsg. von Christa Brüstle, Nadia Ghattas, Clemens Risi und Sabine Schouten, Bielefeld 2005, S. 33-44; Stephanie Jordan, Moving Music. Dialogues with Music in Twentieth-Century Ballet, London 2000; Christine Lubkoll, „Rhythmus. Zum Konnex von Lebensphilosophie und ästhetischer Moderne um 1900", in: dies. (Hrsg.), Das Imaginäre des Fin de siècle. Ein Symposium für Gerhard Neumann, Freiburg i.Br. 2002, S. 83-109. Alle drei Darstellungen verbleiben im kulturwissenschaftlichen Diskurs und blenden die transzendentalphilosophischen Grundfragen des Rhythmus und ihre Neubeantwortung in der empirischen Ästhetik aus.

26 Émile Jaques-Dalcroze, Methode Jaques-Dalcroze, Teil 1: Rhythmische Gymnastik, Bd. 1, Neuchâtel u. a. 1906 (Bd. 2 nicht erschienen); Teil 2: Studium des Notenplanes, Neuchâtel u. a. 1907. Zeitgleich auch auf Frz. erschienen: Der Rhythmus als Erziehungsmittel für das Leben und für die Kunst. Sechs Vorträge von E. Jaques-Dalcroze zur Begründung seiner Methode der rhythmischen Gymnastik, Basel 1907. 
Verità am Lago Maggiore auf, wo sie 1914 auf Mary Wigman treffen, die bereits in München Labans Schülerin war, nachdem sie 1910 bis 1912 in Hellerau studiert hat. In den Jahren des Weltkriegs baut Laban eine Rhythmikschule in Zürich auf, bleibt aber der Künstlerkolonie auf dem Monte Verità verbunden. Er ist hier längst vertraut mit den Zeitphänomenologien Bergsons und Husserls. Dass er die empirischen Ästhetiker gelesen hat, ist nicht belegt und wenig wahrscheinlich; wie die meisten Tänzer und Gymnasten, von Ausnahmen abgesehen, rezipiert er sie aus der zweiten Hand der Lebensphilosophie. Bedeutsam ist für ihn Bergsons These, Bewegtheit sei nicht nur ein Merkmal von Bewusstseinsvorgängen, sondern als élan vital ein Merkmal des Lebens überhaupt. ${ }^{27}$ Bei Laban konkretisiert sich dies theoretisch zunächst im Tanz als Raumkunst. Während, wie die dissidente Jaques-Dalcroze-Schülerin Wigman rückblickend sagt, viele Tänzer des Münchner Fin de Siècle nicht über eine dilettantische Befreiung des Tanzes von der Musik und eine vage Einbettung des Tanzes ins Leben hinausgekommen seien, „überlebendes Symbol des Münchner Faschings“, 28 sei es Laban gelungen, eine genuine, präkulturelle, gerade dadurch lebensdurchtränkte Gesetzmäßigkeit des Tanzes zu finden. „Er schuf für den Tanz das, was die Harmonielehre für die Musik bedeutet: die Raumlehre der Bewegung. "29 Die impliziten Bewegungsdynamiken des gegebenen Raums und des eigenen Körpers werden dem Tänzer als „ihre eigene innere Bewegtheit“ bewusst. ${ }^{30}$ Diese Bewegtheit wird „in sichtbare Körperrhythmik“ transformiert. ${ }^{31}$ Laban setzt also nicht den leeren, abstrakten Raum voraus, sondern den mit Dingen, Architekturen und ihrer spezifischen Räumlichkeit von Lebenszusammenhängen schon gestalteten Raum. In diesem Lebens-Raum strebt der Tänzer zu gegebenen Raumpunkten, erzeugt dadurch Spannung, evoziert Gegenrichtungen (oben, unten, seitlich) und entwirft so die nächste Bewegung. Die Bewegung ist nicht Resultat bestimmter Bedingungen des menschlichen Körpers, noch weniger Ausdruck von Subjektivität. Er ist das Sich-Erstrecken von Körper und Seele in das Da des gegebenen, nun aber zu eigentlichem Dasein bestimmten Raums. In Die Welt des Tänzers, ${ }^{32}$ dem wegweisenden Buch der Tanzbewegung, formuliert Laban das als genau die philosophische Phänomenologie, die mit Heideggers Begriff des Daseins angemessen charakterisiert ist. Er entwirft eine Bewegungsphilosophie, die körperliche Bewegung als Ausdruck der seelischen Bewegtheit zwischen sieben Graden und Arten der Phänomenalität auffasst, in der die Bewusstseinsgegenstände vorliegen. Die Gegenstände sind weder subjektphilosophisch gedacht noch als Objekte, deren naturalem Ansich man sich ausliefert. Sie sind Phänomene, und erst dadurch erschließt sich ihre Lebensdynamik schon gelebten Lebens, dadurch werden sie mit neuem Leben erfüllt. „Wenn der Mensch das bewußt über allen Teilungen des Verstandes stehend erlebt, so ist er Tänzer. "33 Das ist zugleich eine Definition des 'freien Tanzes`, dem Schlagwort der Bewegung um Laban. Mary Wigman nennt ihre Schule in Dresden, gegründet 1920, „Schule des Freien Tanzes“. In ähnlicher Weise sind mehr oder weniger alle großen Namen der Tanzbewegung direkt mit der Lebensphilosophie und mittelbar mit der empirischen Rhythmusforschung verbun-

27 Henri Bergson, L'évolution créatrice, Paris 1907, bes. S. 95ff. Die deutsche Übersetzung besorgte eine Figur des George-Kreises, Gertrud Kantorowicz, Schöpferische Entwicklung, Jena 1921.

28 Mary Wigman, „Rudolf von Labans Lehre vom Tanz“, in: Die neue Schaubühne. Monatshefte für Bühne, Drama und Film 3 (1921), S. 99-106, hier S. 99.

29 Ebd., S. 100.

30 Ebd., S. 103.

31 Ebd., S. 104.

32 Rudolf von Laban, Die Welt des Tänzers, Stuttgart 1920.

33 Ebd., S. 6. Das siebengradige Schema S. 5. 
den: die „Freitanzschule“ der amerikanischen Schwestern Isadora und Elisabeth Duncan (ab $1904)^{34}$, die Palucca-Schule (ab 1925), die Günther-Schule (ab 1923).

Die Figur aus der Tanzbewegung, die ausdrücklich auf die empirische Forschung zurückgreift, ist Rudolf Bode. Er studiert Musik am Leipziger Konservatorium und gleichzeitig Philosophie an Wilhelm Wundts Laboratorium, wo er 1906 mit der Arbeit Die Zeitschwellen für Stimmgabeltöne mittlerer und leiser Intensität promoviert wird. Hier findet Bode zu seiner Lebensfrage, die er dann tänzerisch, gymnastisch und später im NS-Staat kulturpolitisch ausformuliert: Warum fasst der Mensch akustische Reize, die im identischen (im Millisekundenbereich liegenden) Zeitabstand erklingen, einmal als einen, einmal als zwei Töne auf, je nach Tonhöhe? (Die Zeitdistanz, bei der die Wahrnehmung von Reizen zu einem Ton verschmilzt, wird als Zeitschwelle bezeichnet.) Allgemeiner: Warum nimmt der Mensch identische Reizserien, bei denen die Zeitachse manipuliert wird, als Differenz auf der Tonhöhenebene wahr und umgekehrt? 1910-1911 ist Bode Dozent in Hellerau unter Jaques-Dalcroze, absolviert dort selbst 1912. Vor dem Hintergrund seiner intimen Kenntnis der empirischen Rhythmusforschung bei Wundt und der praktischen Erfahrung mit der Methode Jaques-Dalcroze lernt er in diesen Jahren Texte von Ludwig Klages kennen. Welche genau, lässt sich schwer sagen, vielleicht die Studie Ausdrucksbewegung und Gestaltungskraft, 1913 beim Engelmann-Verlag in Leipzig erschienen. In diesem Verlag publizierten auch Wundt und sein Schülerkreis, auch Bodes Dissertation wird hier verlegt. Klages' Studie ist eine der ersten seiner wissenschaftlichen Publikationen nach dem dichterischen Frühwerk und dem George-Buch von 1902, von dem sich Klages nun distanziert. Das Thema Lebensrhythmus übernimmt Klages aus dem George-Buch und überträgt es auf ein anderes Medium, den körperlichen Ausdruck. Diesen fasst Klages als den leiblichen Vollzug der inneren seelischen Bewegtheit, die er zum einen aus einer stabilen Individualität erklärt (die verschiedensten Seelenbewegungen und ihre Ausdrucksgesten erkenne ich als meine Bewegungen), zum anderen mit einer Bewegtheit, die wie eine „Welle“ 35 vom einen Individuum zum nächsten wogt (ich erkenne in meiner Bewegung die Bewegtheit des Anderen). Ähnlich wie Heidegger in den frühen Freiburger Vorlesungen Heideggers, aber einige Jahre eher beschreibt Klages die „spezifische Bewegtheit“36 des Leib-Seele-Ganzen:

„Es entspräche darnach dem Streben schlechtweg die Zentrifugalität, dem Widerstreben die Rückläufigkeit der Bewegung; dem inneren Fortschreiten der Bewegungsfluß, dem Beharren der Bewegungsstillstand, den Widerstands-, Hemmungs- und Spannungsgefühlen jede Funktionsgestaltung, die als gegen physische Widerstände gerichtet befähigt wäre, gesteigerte Kontaktempfindungen hervorzurufen. "37

Solche lebensphilosophischen Überlegungen elektrisieren den Tänzer und Musiker Bode. In Klages' Gegenüberstellung des bewegten Leib-Seele-Ganzen und der fest-stellenden, fixierenden Tätigkeit des Geistes erkennt er die Problemstellung in Hellerau aus neuer Perspektive wieder. Jaques-Dalcrozes Methode erscheint ihm nun als sekundäre

34 Sie waren der Ausgangspunkt für Erich von Hornbostels „Melodischer Tanz. Eine musikpsychologische Studie“ (1903), ed. in: ders., Tonart und Ethos. Aufsätze zur Musikethnologie und Musikpsychologie, hrsg. von Christian Kaden und Erich Stockmann, Leipzig 1986, S. 76-85 (zu Isadora Duncans Tänzen zu Musik von Beethoven und Chopin S. 76). Hornbostel kennt offenkundig die empirische Dauernforschung, Stand 1903, nicht: Er macht psychologische Beobachtungen zur inneren Bewegtheit von Melodieverläufen, ohne ihre unmittelbare psychologische Korrelation zur Wahrnehmung von Dauerngrenzen zu erfassen.

35 Ludwig Klages, Ausdrucksbewegung und Gestaltungskraft, Leipzig 1913, S. 9.

36 Ebd., S. 26.

37 Ebd., S. 27. 
Rhythmisierung abstrakter metrischer Strukturen der Musik, als Geisttätigkeit, also, während doch eigentlich der primäre Rhythmus der leib-seelischen Bewegtheit erfasst werden müsste. Wie Klages selber bringt Bode das zusammen mit den Befunden der empirischen Ästhetik: Fixiert man die Dauernwahrnehmung, so gerät die Tonqualitätswahrnehmung ins Schwimmen; fixiert man die Tonqualitätswahrnehmung, gerät die Dauernwahrnehmung in Fluss. Diese Unschärferelation zwingt zur Annahme, dass am Grund des Wahrnehmens eine konstitutive Bewegtheit herrscht, die es nicht erlaubt, die beiden Dimensionen Dauer und Dauernbegrenzungsklang absolut zu setzen. Wenn schließlich Klages Tanz und Arbeit als „in ebenderselben Lebensnähe“ wie die unmittelbaren Ausdrucksgesten auffasst, ${ }^{38}$ erkennt Bode seine Mission: eine theoretische Grundlegung der körperlichen Bewegungslehre auf dem Fundament von Klages' Begriff des leib-seelischen Rhythmus. ${ }^{39}$ Er wendet sich von Jaques-Dalcroze $\mathrm{ab}^{40}$ und gründet 1911 in München die Bode-Schule, die „Lehrgänge für rhythmische Gymnastik" anbietet, aber auch Musikkurse, die freilich ebenso wie Tanz und Gymnastik vom Grundgedanken des Rhythmischen getragen werden. 1912 wird der BodeBund gegründet, der Bode-Kurse in zahlreichen deutschen Städten organisiert. Von allen Protagonisten der Tanzbewegung nimmt damit Bode die Rolle des Cheftheoretikers, im Nationalsozialismus auch des Chefideologen ein. ${ }^{41}$

\section{Jugendbewegung und Reformpädagogik}

Die engen Verflechtungen zwischen der Rhythmusbewegung und der zeitgleich aufkommenden Jugendbewegung und Reformpädagogik und die katalytische Rolle der empirischen Rhythmusforschung können nur angedeutet werden. Einige Rhythmus- und Gymnastikschulen, die von Figuren der Tanzbewegung gegründet werden, sind von vornherein als allgemeinbildende Schulen konzipiert. Der Gedanke des Rhythmischen in der leib-seelischen Bewegtheit des Menschen taugt als pädagogisches Paradigma. Die Duncan-Schule in Darmstadt und Jaques-Dalcrozes Institut in Hellerau bzw. nach dem Weltkrieg Laxenburg bei Wien, sind Beispiele. Umgekehrt arbeiten in den Reformschulen, die sich schon vor dem Krieg gründen, viele Lehrer, die mit der Rhythmusbewegung verbunden sind, etwa die Musikwissenschaftler Ernst Kurth und August Halm in der Freien Schulgemeinde Wickersdorf, der Laienmusiker Fritz Jöde und der Laientänzer Max Tepp an jenen Hamburger Volksschulen, an denen reformpädagogische Konzepte probiert werden. Von Kurth, Halm und Jöde wiederum adaptiert Wyneken in seinen weltanschaulichen Texten zur Reformpädagogik den Begriff der Energie, der mit dem des Rhythmus eng verwandt ist. ${ }^{42}$ Christine Baer-Frisell, Rhythmuslehrerin in Laxenburg, gibt in den 1920er Jahren Ferienkurse für Lehrer an Montessori-Schulen. Martin Luserke, erst Gefolgsmann von Hermann Lietz am Landerziehungsheim Haubinda, dann von Wyneken

38 Ebd., S. 86.

39 Rudolf Bode, Rhythmus und Körpererziehung. Fünf Abhandlungen, Jena 1923; ders., Musik und Bewegung, Kassel 1930, Berlin 21942. Vgl. eine Vielzahl weiterer Schriften Bodes bis 1933, die in der Substanz immer den beschriebenen Gedanken enthalten.

40 Und begründet dies öffentlich in Rudolf Bode, Das rhythmische Problem und die Methode JaquesDalcroze, Leipzig 1914.

41 Aus den völkisch orientierten Schriften Rudolf Bodes ab den späten 1920er Jahren seien genannt: „Leben, Rhythmus, Volkstum“, in: Der Wettkampf 5 (1928), S. 337-341; Bewegung und Gestaltung. Von den Kulturaufgaben der körperlichen Erziehung, Berlin 1936.

42 Gustav Wyneken, Weltanschauung, München 1940 (geschrieben 1936), passim. 
in Wickersdorf, schließlich Gründer der Schule am Meer auf Juist, pflegt Verbindungen zur Tanzbewegung. Ludwig Klages hält beim Freideutschen Jugendtag 1913 auf dem Hohen Meißner eine Rede. Bode wird 1933 eine wichtige Figur der NS-Bildungspolitik aus dem Geist der Reformpädagogik. Die Liste könnte verlängert werden. Mit guten Gründen deutet Oelkers sogar die Künstlerkolonie auf dem Monte Verità als experimentelle Spielart der Reformschule. ${ }^{43}$ Angedeutet seien schließlich die Verzahnungen des George-Kreises mit der Jugendbewegung, auch der Jugendmusikbewegung. ${ }^{44}$

Die Aufzählung könnte den schiefen Eindruck erwecken, Jugendbewegung und Reformpädagogik empfingen die Erkenntnisse der empirischen Rhythmusforschung bloß aus zweiter oder dritter Hand. Die Sachlage ist komplexer. Zum einen gibt es mit Ernst Meumann eine herausragende Figur der Reformpädagogik, die in den Labors der empirischen Rhythmusforschung ihre Lehr- und Meisterjahre verbracht hat. Meumann, mittlerweile Professor in Hamburg, ist Mitbegründer des „Allgemeinen deutschen Verbands für Erziehungs- und Unterrichtswesen“, der Organisation aller reformorientierten Pädagogen in Deutschland innerhalb und außerhalb der Landerziehungsheime. Er organisiert zwischen 1911 und 1913 die großen pädagogischen Kongresse, die der Reformpädagogik entscheidende Impulse geben. Zum anderen sollte man nicht das empirische Moment geringschätzen, das im phänomenologischen Ansatz der Philosophen (Bergson, Klages, Dewey) und der Tanzkünstler (Duncan, Laban, Bode, Günther), die für die Reformpädagogik maßstäblich werden, selber liegt.

\section{Musiktheorie}

In der Musiktheorie hat das Thema Rhythmus eine lange Tradition, zurückgehend auf Aristoxenos' Elementa rhythmica und Augustins De musica, letztere eine reine Metrumslehre der (gesungenen) Poesie. Im Mittelalter wird die Lehre von den musikalischen Dauern zu einem Thema der mensurierenden Notation. Metrum und Rhythmus als solche werden dort nicht mehr verhandelt, bleiben aber in den Notationskonzepten unterschwellig wirksam. ${ }^{45}$ Vollends aus dem Blick geraten die musikalischen Dauern in der Musiktheorie vom 17. bis zum 19. Jahrhundert. Dann reift allmählich die Einsicht, dass sich Tonverbindungen (Melodik) und Klangverbindungen (Harmonik) in der Zeit ereignen und die Zeitstruktur auf die Gesetzmäßigkeiten dieser Verbindung Einfluss nimmt. ${ }^{46}$ Dass die zeitliche Ordnung der Poesie dafür nicht mehr der Maßstab sein kann, wie von der Antike bis noch ins frühe 18 . Jahrhundert, wird von allen Musiktheoretikern gesehen. ${ }^{47}$ Konsequent alle Anklänge an die Sprachmetrik abzuschütteln ist man indessen noch nicht in der Lage. Als Hürde erweist sich

43 Jürgen Oelkers, Reformpädagogik. Eine kritische Dogmengeschichte, Weinheim 1989, 31996, ${ }^{4} 2005$, S. 319 ff.

44 Vgl. Dieter Martin, „,Wer je die Flamme umschritt'. Musik am Lagerfeuer“, in: Realität als Herausforderung. Literatur in ihren konkreten historischen Kontexten, hrsg. von Ralf Bogner, Ralf Georg Czapla, Robert Seidel und Christian von Zimmermann, Berlin 2011, S. 427-446.

45 Für die Modalnotation des 12. und 13. Jahrhunderts vgl. Rainer Bayreuther, Untersuchungen zur Rationalität der Musik in Mittelalter und Früher Neuzeit, Bd. 1: Das platonistische Paradigma. Untersuchungen zur Rationalität der Musik vom 12. bis zum 16. Jahrhundert, Freiburg i. Br. 2008, $\$ 16$ und passim.

46 Moritz Hauptmann, Die Natur der Harmonik und der Metrik. Zur Theorie der Musik, Leipzig 1853.

47 Selbst von dem Altphilologen Rudolf Westphal, Elemente des musicalischen Rhythmus mit besonderer Rücksicht auf unsere Opernmusik, Jena 1872 - ein Versuch, mit aristoxenischer Rhythmuslehre die zeitgenössische Musik zu erklären. 
die unklare Abgrenzung von Rhythmus und Metrum. Diese Hürde wird bei Hugo Riemann um 1900 erst in dem Moment genommen, in dem die musiktheoretische Dauernlehre auf das Fundament der empirischen Forschung zur Dauernwahrnehmung gestellt wird. Dies geschieht noch abseits und neben der sich erst formierenden Rhythmusbewegung. In der Theoretikergeneration nach Riemann aber schlägt sich die sachliche Nähe dieser neuen Rhythmustheorie zur Rhythmusbewegung auch in einer personellen und institutionellen Verzahnung nieder.

Betrachten wir zunächst Riemann. Früh, in seiner Dissertation Ueber das musikalische Hören (1873), beschäftigt er sich mit der experimentellen Psychoakustik, hier mit Helmholtz' Lehre von den Tonempfindungen. Ab 1895 arbeitet Riemann in Leipzig und ist mit Wundt persönlich bekannt. ${ }^{48}$ Im Buch Musikalische Dynamik und Agogik (Hamburg 1884) verbleibt er innerhalb des Theorierahmens, den schon Hauptmann und Westphal haben, das Metrum als die Akzent-, den Rhythmus als die Bewegungsstruktur der Musik aufzufassen. Die musikalische Phrase, so konstatiert Riemann, hat innere Strebekräfte, die mit der metrischen Struktur mal zusammenfallen, mal sich gegenpolig zu ihr verhalten. Dass aber eine solche Rhythmustheorie in einer metrischen Theorie der musikalischen Dauer aufgeht, sieht man, wenn man sich die körperbezogenen Ursprungserklärungen ansieht, die diese Auffassung des musikalischen Rhythmus begleiten. Von Karl Philipp Moritz über August Wilhelm von Schlegel bis zu Hausegger ${ }^{49}$ wurden der bilaterale Körperbau des Menschen, das Hin und Her von Atmung und Blutzirkulation, der Doppelschlag des Herzens und anderes als letzter Grund dafür angeführt, dass das Zweiermetrum mit MM. $\approx 60$ das naturgegebene Urmetrum sei. Die Körperlichkeit generiert den Schritt, den Pes, die Bilateralität des Körpers generiert das Metrum. Der Bewegungsaspekt der Musik wäre, auch wo er sich irregulär zum Metrum verhält, auf Schritte und Schrittfolgen zurückgeführt. Diese latent metrische Erklärung alles Rhythmischen durchbricht Riemann erst, als er die Forschung zur Dauernwahrnehmung in Wundts Laboratorium zur Kenntnis nimmt. In der vierten Auflage seines Musik-Lexikons ${ }^{50}$ referiert er Wundts Einsicht, dass eine Abfolge regelmäßiger akustischer Reize als Kette von Gliedern wahrgenommen wird. Der aktuelle Reiz wird mit dem vorhergehenden verbunden und als Vorbereitung für den nachfolgenden aufgefasst. Mit dieser Einsicht ist die alte Auffassung, Rhythmus sei die Bewegung von einer gegebenen Akzentstufe zur nächsten, nicht mehr vereinbar. Die Kausalität kehrt sich um: Indem in der Bewegung von einem Begrenzungsreiz zum nächsten die Reize zu Gliedern einer Kette erst gemacht werden, wird durch die Bewegung die Metrik der Reizabfolge überhaupt generiert. Primär ist also die Bewegung von Reizpunkt zu Reizpunkt, sekundär und von ihr verursacht das Hineindeuten einer bestimmten (`metrischen`) Struktur der Reizpunktserie. Jetzt erst ist die Trennung von Rhythmus und Metrum bei ihrer philosophischen Pointe angekommen. Bei Rhythmus und Metrum handelt es sich nicht einfach um zwei Prädikate, die die Zeitlichkeit unterschiedlicher Aspekte des musikalischen Satzes beschreiben, indem sie die Aspekte als schlichtweg gegeben, Heidegger würde sagen: als vorliegendes Seiendes auffassen. Ohne dass Riemann einen klaren ontologischen Begriff davon hat, findet er heraus, dass Rhythmus und Metrum nicht nur Eigenschaften unterschiedlicher Typen von Entitäten sind, sondern ontologisch kategorial getrennten Sphären zugehören: die Eigenschaft des

48 Aus dem verlorenen Briefwechsel ist ein Brief Riemanns vom 16. August 1907 erhalten, in dem er Wundt zum 75. Geburtstag gratuliert (Univ.-Archiv Leipzig, NA Wundt/III/1601-1700/1603/43/125-126).

49 Friedrich von Hausegger, Die Musik als Ausdruck, Wien 1885.

50 Hugo Riemanns Musik-Lexikon. Theorie und Geschichte der Musik, die Tonkünstler alter und neuer Zeit mit Angabe ihrer Werke, nebst einer vollständigen Instrumentenkunde, Leipzig ${ }^{1} 1882,{ }^{4} 1894$, hier S. 683. 
Metrums dem Seienden, die Eigenschaft des Rhythmus dem Existieren. Riemann setzt auf Grundlage seiner bahnbrechenden Einsicht mit der Theorie von Rhythmus und Metrum noch einmal neu an und verfasst das „System der musikalischen Rhythmik und Metrik“ ${ }^{11}$, in dem mit dem Rhythmus als dem Elementaren begonnen wird und die musikalischen Metren im zweiten Teil als komplexere und großräumigere Bildungen „aus elementaren Rhythmen“ behandelt werden: „nicht ein von der Sprache abstrahiertes Maß für den Zeitverlauf, sondern ein durch rein musikalisch oder noch allgemeinere, psychologisch begründete Postulate sich ergebendes “52. Das kompositorische Postulat des psychologisch begründeten Maßes ist das Motiv: „die musikalische Phrase [ist] die einzelne Geberde des musikalischen Affekts. " ${ }^{53}$ Diese im psychologischen Sinn elementar rhythmische Auffassung des musikalischen Motivs wandert direkt in die Tanzbewegung; bei Bode findet sie sich in genau dieser Weise. ${ }^{54}$

Ich erläutere dies ausführlicher, weil hier der systematische Kern der Problemstellung berührt ist. Für die Musikwissenschaft des 20. Jahrhunderts, sofern sie sich des Themas selten genug überhaupt angenommen hat, war es offenkundig schwierig, ihn festzuhalten. Die meisten fallen, auch dort, wo sie glauben, an Riemann anzuknüpfen, wieder in das Nebeneinander von Rhythmus und Metrum zurück. Der beinahe einzige, ${ }^{55}$ der Wundts und Riemanns Weg weitergeht und den kühnen Versuch unternimmt, alle Topoi der Musiktheorie auf das Existential der Bewegung zu gründen, ist Ernst Kurth. Kurths konkrete Referenzen sind nicht die Leipziger Empiriker und auch nicht primär Riemann. In Wien, wo er ab 1904 Musik und Philosophie studiert, hört er die am Pragmatismus und an der empirischen Psychologie geschulten Friedrich Jodl ${ }^{56}$ und Wilhelm Jerusalem. ${ }^{57}$ Namentliche Erwähnung findet Theodor Lipps, ${ }^{58}$ der die Ästhetik auf den psychischen Zustand der Lust bzw. Unlust und ihre Strebewirkung zurückführt. „Fluß“ und „Bewegung“, die eine „innere ,Rhythmik“" haben, sind die psychischen Grundmodi, aus denen Lipps die ästhetischen Kategorien ableitet. ${ }^{59}$ Das ist der Hintergrund von Kurths Begriff der Energie, aus dem er nun alle Topoi der Musiktheorie entwickelt, auch Harmonielehre ${ }^{60}$ und Kontrapunkt. $^{61}$

Man kann die mit Kurth eingeschlagene Richtung eine Musiktheorie aus dem Geist der Jugendbewegung und der Reformpädagogik nennen. Becking rekurriert mit Herman Nohl auf einen der wichtigsten Stichwortgeber der Reformpädagogik. Beckings Rhythmustheorie ist aufgrund ihrer jugendbewegten Orientierung an Bewegungsmustern des menschlichen

51 Hugo Riemann, System der musikalischen Rhythmik und Metrik, Leipzig 1903.

52 Ebd., S. VIIf.

53 Ebd., S. VIII.

54 Rudolf Bode, Musik und Bewegung, Kassel 1930, Berlin ${ }^{2} 1942$, S. 15.

$55 \mathrm{Zu}$ nennen wäre noch der Riemann-Schüler Gustav Becking, Der musikalische Rhythmus als Erkenntnisquelle, Erlangen 1922, Augsburg 1928.

56 Friedrich Jodl, Lehrbuch der Psychologie, Stuttgart 1897, ${ }^{4} 1916.2$ Bde.

57 Wilhelm Jerusalem, Wege und Ziele der Ästhetik, Wien 1905.

58 Ernst Kurth, Musikpsychologie, Bern 1931, S. 50 und S. 59.

59 Theodor Lipps, Grundlegung der Ästhetik, Hamburg und Leipzig 1903; ders., Die ästhetische Betrachtung und die bildende Kunst, Hamburg und Leipzig 1906 (=2. Teil der Ästhetik), hier 2. Teil, S. 9.

60 Ernst Kurth, Die Voraussetzungen der theoretischen Harmonik und der tonalen Darstellungssysteme, Bern 1913.

61 Ernst Kurth, Grundlagen des linearen Kontrapunkts. Einführung in Stil und Technik von Bach's melodischer Polyphonie, Bern 1917. 
Körpers in der Nachkriegsmusikwissenschaft auf große Skepsis gestoßen, ${ }^{62}$ ein Urteil, zu dem zumindest zu sagen ist, dass es ohne die Würdigung des kulturellen Kontexts von Jugendund Rhythmusbewegung zustande kam. Kurth ist für kurze Zeit Musiklehrer an Wynekens Freier Schulgemeinde Wickersdorf, bevor er dort von einem anderen Musikwissenschaftler, August Halm, abgelöst wird. Seine Berner Habilitationsschrift über die Harmonik ${ }^{63}$ ist teilweise in Wickersdorf entstanden, der Begriff der musikalischen Energie von der Rhythmusauffassung der Reformpädagogik inspiriert und dann mit Lipps theoretisch unterlegt. Kurth bekundet später selbst, seine gesamte musikwissenschaftliche Arbeit sei von den Erfahrungen in Wickersdorf geprägt. ${ }^{64}$ Man kann berechtigte Skepsis haben, ob Kurth den Ansatz, im Rhythmus das Element aller musikalischen Parameter zu sehen, musiktheoretisch sauber eingelöst hat.

Die jugend- und rhythmusbewegte Musikforschung im frühen 20. Jahrhundert hat die bleibende Einsicht erbracht, dass vor jeder musiktheoretischen und musikgeschichtlichen Arbeit eine musikphilosophische Klärung stehen muss, was Dauer und Rhythmus in der Musik sind. Die Einsicht war erst durch Erkenntnisse der empirischen Dauern- und Rhythmusforschung möglich geworden. Dass nicht nur die Forschungserträge der historischen Musikforschung auf dem Feld des Rhythmus seitdem gering geblieben sind, sondern auch keine nennenswerte transdisziplinäre Forschung zu den vielen Phänomenen wie Sport, Tanz, Poesie stattgefunden hat, in denen jene akustische Dauernbegrenzung auftritt, von der die empirische Rhythmusforschung eine elementare Prädisziplinarität schon um 1900 belegt hat, muss zu denken geben.

62 Etwa Wilhelm Seidel, Über Rhythmustheorien der Neuzeit, Bern und München 1975. Zur weiteren musikwissenschaftlichen Rezeption Beckings vgl. Martin Pfleiderer, Rhythmus. Psychologische, theoretische und stilanalytische Aspekte populärer Musik, Bielefeld 2006, S. 123f.

63 Kurth, Die Voraussetzungen (wie Anm. 60).

64 Dokumentiert bei Hilmar Höckner, Die Musik der Jugendbewegung, Wolfenbüttel 1927, S. $93 \mathrm{f}$. 